\title{
In reply: Comment on the editorial relating to: Transversus abdominis plane block compared with wound infiltration for postoperative analgesia following Cesarean delivery: a systematic review and network meta-analysis
}

\author{
Vishal Uppal, MBBS, MSc, FRCA (1) - Garrett Barry, MD
}

Received: 17 November 2020/ Accepted: 18 November 2020/Published online: 6 January 2021

(C) Canadian Anesthesiologists' Society 2021

\section{To the Editor,}

Sultan et al. have expressed some concerns regarding our pragmatic interpretation of their study in our recent editorial entitled "Management of pain after Cesarean delivery without intrathecal morphine: Networking for the best answer." ${ }^{1,2}$ Sultan et al. believe the take-home message from their network meta-analysis should be that some intervention is better than no intervention. Nevertheless, we believe that clinicians should strive for excellence by opting for their patients' best option. In this regard, transversus abdominis plane (TAP) blocks ranked highest not only for the primary outcome (24-hr opioid use) but also for feasibility and multiple other patient-centred outcomes, including 12- and 24-hr rest pain, postoperative nausea and vomiting, and time to first analgesic request. We want to clarify the rationale for our interpretation of their study results for the journal readers.

Sultan et al. assert that "wound infiltration (WI) should be offered to women for analgesia after Cesarean delivery in the absence of long-acting neuraxial opioids, if TAP blocks or wound catheters (WC) are not available options." Assuming either lack of training in an ultrasound-guided technique or ultrasound equipment itself, the landmarkguided double-pop TAP block described by McDonnell et al. is simple, effective, and requires no extra resources than what would be used for WI. ${ }^{3}$ Furthermore, a surgeonplaced TAP block is another equally effective alternative. ${ }^{4}$

This reply letter is related to letter 20-01151.

V. Uppal, MBBS, MSc, FRCA $(\bowtie) \cdot$ G. Barry, MD

Department of Anesthesia, Perioperative Medicine and Pain

Management, Dalhousie University, Nova Scotia Health

Authority and IWK Health Centre, Halifax, NS, Canada

e-mail: v.uppal@dal.ca
As highlighted in our editorial, there are multiple inherent limitations of catheter techniques compared with a singleshot technique that are hard to argue against.

Their data show that inactive controls (IC) were inferior to all active comparisons except WI for the primary outcome. No statistically significant difference was observed between the WI and IC groups. Sultan et al. propose that there may be a clinical difference between WI and IC in the absence of statistical difference in favour of WI. Nevertheless, the same could be argued for the difference between TAP block and WI, which shows a large effect size in favour of TAP blocks. Furthermore, their study failed to show any statistical difference between active interventions-as per the popular aphorism, "absence of evidence is not evidence of absence." In other words, the data are likely to be underpowered to detect the difference between the active comparators as evident by a wide $95 \%$ confidence interval for standard mean deviation values. The Cochrane collaboration recommends against making any firm conclusion in such situations. $^{5}$

We agree with the authors that the surface under the cumulative ranking curve (SUCRA) has its limitation and should not be used in isolation. Nevertheless, it provides "a simple transformation of the mean ranks, is useful in providing a hierarchy of the treatments, and accounts both for the location and the variance of all relative treatment effects." ${ }^{6}$ Furthermore, clustering of SUCRA values can aid in analysing multiple outcomes. For example, the Figure shows a clustered ranking plot of the SUCRA values for 24-hr opioid consumption and 24-hr rest pain scores. As "a picture is worth a thousand words", we will let readers look and decide for themselves the best option for postoperative analgesia after Cesarean delivery in the absence of long-acting neuraxial opioids. Our view is that 


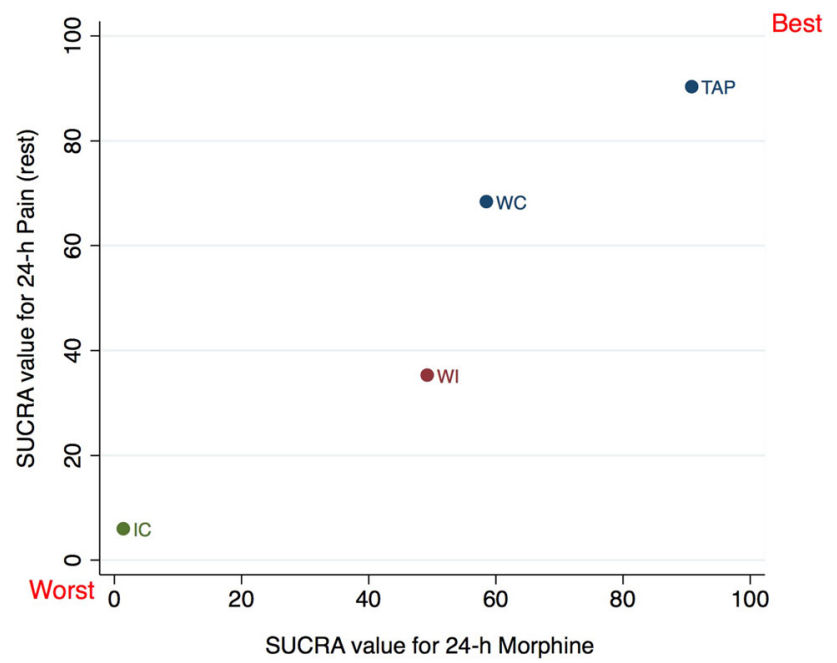

Figure Clustered ranking plot for the outcomes 24-hr morphine consumption and 24-hr pain score at rest. This method performs statistical clustering of treatment groups based on their surface under the cumulative ranking curve (SUCRA) values, which shows transversus abdominus plane (TAP) block and wound catheter (WC) clustered together as the best treatments (blue), followed by wound infiltration (WI) then inactive control. A higher SUCRA value indicates a better-ranked intervention. The top-right position on the plot indicates the best treatment, and the bottom-left indicates the worst treatment for the combination of the two plotted outcomes

clinicians should aim for the best, not for "something is better than nothing."

Disclosures None.
Funding statement None.

Editorial responsibility This submission was handled by Dr. Hilary P. Grocott, Editor-in-Chief, Canadian Journal of Anesthesia.

\section{References}

1. Barry G, Uppal V. Management of pain after cesarean delivery without intrathecal morphine: networking for the best answer. Can J Anesth 2020; DOI: https://doi.org/10.1007/s12630-020-01819-w.

2. Sultan P, Carvalho B, Halpern SH. Comment on the editorial relating to: Transversus abdominis plane block compared with wound infiltration for postoperative analgesia following cesarean delivery: a systematic review and network meta-analysis. Can J Anesth 2021; DOI: https://doi.org/10.1007/s12630-020-01872-5.

3. McDonnell JG, Curley $G$, Carney J, et al. The analgesic efficacy of transversus abdominis plane block after cesarean delivery: a randomized controlled trial. Anesth Analg 2008; 106: 186-91.

4. Narasimhulu DM, Scharfman L, Minkoff H, George B, Homel P, Tyagaraj $K$. A randomized trial comparing surgeon-administered intraoperative transversus abdominis plane block with anesthesiologist-administered transcutaneous block. Int J Obstet Anesth 2018; 35: 26-32.

5. Higgins J, Thomas J, Chandler J, et al. Cochrane Handbook for Systematic Reviews of Interventions, $2^{\text {nd }}$ Edition (section 15.6.4). John Wiley \& Sons, Chichester UK; 2019.

6. Chaimani A, Higgins JP, Mavridis D, et al. Graphical tools for network meta-analysis in STATA. PLoS One 2013; DOI: https:// doi.org/10.1371/journal.pone.0076654.

Publisher's Note Springer Nature remains neutral with regard to jurisdictional claims in published maps and institutional affiliations. 